\title{
Review of endocrine complications in adult patients with $\beta$-thalassaemia major
}

\author{
Ploutarchos Tzoulis \\ UCL Medical School, Royal Free Hospital, London, UK
}

\begin{abstract}
Endocrine abnormalities are amongst the most common complications of $\beta$-thalassaemia major (TM). This is an overview of endocrinopathies of adult patients with $\beta$-thalassaemia major, excluding osteoporosis and fertility issues. This review will focus on emerging evidence in the last 5 years with regards to endocrinopathies in patients with TM.
\end{abstract}

\section{Prevalence}

In light of the significant increase of life expectancy of patients with TM in recent years, endocrinopathies have become even more topical. The prevalence of various endocrine disorders varies significantly between different studies. Numerous studies suggest that more than half of patients with TM, even in paediatric cohorts, have at least 1 endocrinopathy. ${ }^{1-6}$ The most recent study by Ang et al., including 92 adult patients with TM (median age 36 years) from the largest UK thalassaemia unit, showed much higher prevalence rates of endocrine disorders than previous studies. In this cohort, $75 \%$ of patients had at least one endocrinopathy, 67\% had hypogonadism, $41 \%$ diabetes mellitus (DM), 17\% hypoparathyroidism and $14 \%$ hypothyroidism. ${ }^{1}$ This difference was attributed to the much older age of this patient cohort in comparison to previous studies. ${ }^{1}$ This study, in keeping with other studies, ${ }^{2}$ showed also that increasing age is an independent risk factor associated with DM and hypogonadism. A past study, conducted in the same major thalassaemia unit in the UK in 1997, included 97 patients with mean age of 24 years and reported that $66 \%$ of patients had hypogonadism, $20 \% \mathrm{DM}, 13 \%$

Correspondence: Ploutarchos Tzoulis, UCL Medical School, Royal Free Hospital, London, UK

E-mail: ptzoulis@yahoo.co.uk

Key words: endocrine abnormalities; $\beta$-thalassaemia; hypogonadism; endocrinopathies.

(C) Copyright P. Tzoulis, 2014

Licensee PAGEPress, Italy

Thalassemia Reports 2014; 4:4871

doi:10.4081/thal.2014.4871

This article is distributed under the terms of the Creative Commons Attribution Noncommercial License (by-nc 3.0) which permits any noncommercial use, distribution, and reproduction in any medium, provided the original author(s) and source are credited. hypoparathyroidism and 10\% hypothyroidism. ${ }^{3}$ It also demonstrated a clear association between genotype in TM major and hypogonadism as well as DM. ${ }^{3}$ Recent data of the French National Registry for $\beta$-TM showed that $55.2 \%$ of patients older than 24 years had hypogonadism, $11.2 \%$ diabetes and $18.0 \%$ hypothyroidism. ${ }^{2}$ The French Registry also found that the frequency of endocrine organ damage related to iron overload increased with age, with the occurrence of complications being rare in childhood. ${ }^{2}$ The largest study of prevalence of endocrine complications, including 3,817 patients with TM in 29 countries, showed lower prevalence rates than the recent UK and French data such as $40.5 \%$ for hypogonadism, $9.9 \%$ for glycaemic abnormalities, $6.9 \%$ for hypoparathyroidism and $3.2 \%$ for hypothyroidism. ${ }^{7}$ However, this large multi-centre study included also a large proportion (36\%) of patients under the age of 16 .

Longitudinal studies have reported that the prevalence of endocrine complications has declined in the last few decades thanks to more effective iron chelation and earlier age of first exposure to chelation treatment. ${ }^{8}$ However, recent studies ${ }^{1,2}$ have indicated increase in the prevalence rates of endocrinopathies as the mean age of cohorts and life expectancy have risen significantly. Overall, the prevalence of endocrine disorders in TM patients is affected by various factors such as age, degree and type of chelation, compliance with chelation, age of first exposure to chelation therapy, age of first transfusion, haemoglobin level attained before blood transfusion and genotype of TM.

\section{Predictors / pathophysiology of endocrinopathies}

Iron toxicity has been regarded as the most likely cause of development of endocrine disorders in patients with TM. A combination of pathologies, including direct tissue damage from iron deposition, damage from chronic anaemia, chelation of other essential elements such as zinc, direct toxicity of non-transferrin-bound iron through reactive oxygen species formation contribute to the development of endocrinopathies in this context.

A recent retrospective study ${ }^{1}$ on the associations between common endocrinopathies and iron load parameters (longitudinal data over a mean of 8 years) demonstrated that:

- There was a strong association between a history of myocardial T2 $<20 \mathrm{msec}$ and hypogonadism as well as DM in keeping with similar findings in a cross-sectional study by Au et al. which also showed strong association of low myocardial T2 with hypoparathyroidism and hypothyroidism. ${ }^{9}$ Patients with hypogonadism and DM had median myocardial T2 values of $12.6 \mathrm{msec}$ and 12.3 msec compared with $23.8 \mathrm{msec}$ and $24.8 \mathrm{msec}$ respectively in patients without those complications. Myocardial T2 $<20$ msec was strongly associated with the occurrence of DM [OR 19.3; 95\% CI 4.3 - 86.7; P < 0.001] and hypogonadism [OR 3.9; 95\% CI $1.4-10.5 ; \mathrm{P}=0.008]^{1}$ 
- Increasing age was associated with DM and hypogonadism. Each 1 year increase in age was associated with 1.1 increased odds of developing DM [95\% CI $1.0-1.2 ; \mathrm{P}=0.002]$ and hypogonadism [95\% CI $1.0-1.2 ; \mathrm{P}=0.016]$. $^{1}$

- Average 10-year serum ferritin levels > $1250 \mathrm{mcg} / \mathrm{l}$ and $>2000 \mathrm{mcg} / \mathrm{l}$ were significantly associated with DM [OR 14.8; 95\% CI $2.4-90.0$; $\mathrm{P}=0.003$ ] and hypogonadism [OR 2.9; 95\% CI $1.0-8.3 ; \mathrm{P}=0.047$ ] respectively. ${ }^{1}$ Some studies, but not all, have identified serum ferritin as a prognostic marker for progression to endocrine dysfunction. ${ }^{10,11}$

- Liver T2 values as well as LIC (liver iron concentration) by MRI-R2 (Ferriscan) values were not associated with the development of endocrinopathies.

Therefore, myocardial iron loading is a good surrogate marker for significant iron overload in the pancreas and pituitary gland, as also shown in other studies which suggested that iron loading in the pituitary and pancreas precedes myocardial iron loading. ${ }^{12-14}$ Myocardial T2 $<20 \mathrm{msec}$ is a good predictor of significant and prolonged iron overload in the pituitary and pancreas. Abnormal myocardial T2 should prompt intensification of iron chelation therapy and investigation and close monitoring for endocrinopathies.

\section{Reversibility of endocrine complications}

Endocrine complications of TM were in the past regarded, in gener$\mathrm{al}$, irreversible. This concept has been recently challenged since there is high quality evidence from a randomised trial by Tanner et al. that combined therapy with desferrioxamine (DF0) and deferiprone (DFP) compared to desferrioxamine monotherapy can improve cardiac iron loading and cardiac function (an increase by $3 \%$ in ejection fraction over one year)..$^{15}$

A study by Farmaki et al., including 52 patients with a 5-7 year follow-up, demonstrated the efficacy of combined chelation therapy with DFO and DFP in reducing total body iron load and reversing as well as preventing endocrine complications. Patients on combination therapy had a statistically significant reduction of the total body iron load in comparison to previous DFO monotherapy, as indicated by ferritin, cardiac and liver iron. The proportion of patients with abnormalities in glucose handling such as DM, impaired glucose tolerance (IGT) and impaired fasting glycaemia (IFG) was reduced from $78 \%$ to $34 \% .{ }^{16}$ Amongst 18 patients on thyroxine, 10/18 managed to discontinue thyroxine and 4/18 reduced the dose. Thus, intensive chelation therapy reversed most cases of compensated or subclinical hypothyroidism and also prevented any new cases of hypothyroidism. In addition, $50 \%$ of male hypogonadal patients achieved normal testosterone levels and were able to discontinue testosterone replacement therapy. Therefore, patients receiving intensive combined chelation reversed and prevented multiple endocrine complications through decrease of iron load which had accumulated over an extensive period. ${ }^{16}$

A different study by the same group ${ }^{17}$ assessed the effect of enhanced iron chelation treatment on glucose metabolism in patients with b-TM major. 41 patients, previously treated with DF0 only, were switched to combined treatment with DFO and DFP. At baseline, 6/41 had DM, 15/41 IGT, 6/41 IFG and 14/41 had normal glucose handling. Combination therapy markedly reduced ferritin levels from $2991 \pm 2093$ $\mathrm{mcg} / \mathrm{l}$ to $638 \pm 1345 \mathrm{mcg} / \mathrm{l}(\mathrm{P}<0.001)$. Glucose responses were improved at all times during an oral glucose tolerance test (OGTT), particularly in patients in early stages of glucose intolerance. Out of 21 patients having at baseline IGT or IFG, more than half (12/21) restored normal glucose handling. Insulin secretion increased markedly in the overall group of patients, mainly due to the increases in insulin secretion in the IGT patients. Insulin sensitivity declined in all groups of patients, but this difference did not reach statistical significance. In total, the most significant improvement in glucose disturbances were found in patients with IGT and IFG. ${ }^{17}$

Another study, examining the effect of intensive chelation on endocrinopathies, found that intensive chelation therapy reversed hypothyroidism, while it did not have positive effect on glycaemic status or gonadal function. After a 4-year observational period, patients who developed new endocrine/metabolic complications were switched from DFO monotherapy to a combined intensive iron chelation regimen of DFO + DFP. After 24 months of intensive chelation with subsequent reduction of median ferritin from $1500 \mathrm{mcg} / \mathrm{l}$ to $569 \mathrm{mcg} / \mathrm{l}(\mathrm{P}<$ $0.001), 8 / 12$ patients on thyroxine were able to discontinue it and $4 / 12$ reduced their thyroxine dose. ${ }^{11}$

A multi-centre retrospective cohort study, published in 2014, examined the incidence and progression of endocrine disorders during longterm treatment with deferasirox (Exjade) in a real clinical practice setting. This cohort included 86 patients with TM treated with once daily Exjade for a median duration of 6.5 years. The incidence of new endocrine complications was only $7 \%$ and included 5 new cases of hypogonadism and 1 new case of hypoparathyroidism. No new cases of diabetes or hypothyroidism were recorded. These findings suggested a low rate of new endocrine disorders and stabilisation of pre-existing disorders such as diabetes and hypothyroidism. ${ }^{18}$

\section{Glucose metabolism}

The prevalence of diabetes mellitus (DM) in patients with $\beta$-TM has been estimated to be between 3.2 and $41 \% .1,2,7,19$ Abnormalities in glucose handling are relatively common complications in patients with TM. According to most recent diagnostic criteria published by American Diabetes Association in 2010, different states in glucose metabolism based on 75-gr oral glucose tolerance test (OGTT) are classified into:

- Diabetes (DM): Fasting plasma glucose (FPG) $\geq 7.0 \mathrm{mmol} / \mathrm{l}(\geq 126$ $\mathrm{mg} / \mathrm{dl}$ ) or 2 -hour plasma glucose $\geq 11.1 \mathrm{mmol} / \mathrm{l}(\geq 200 \mathrm{mg} / \mathrm{dl})$

- Impaired Glucose Tolerance (IGT): 2-hour plasma glucose 7.8 - 11.0 $\mathrm{mmol} / \mathrm{l}(140$ - $199 \mathrm{mg} / \mathrm{dl})$

- Impaired Fasting Glycaemia (IFG): FPG 5.6 - 6.9 mmol/ (100 - 125 $\mathrm{mg} / \mathrm{dl})$

- Normal glucose metabolism: FPG $<5.6 \mathrm{mmol} / /(<100 \mathrm{mg} / \mathrm{dl})$ and 2 hour plasma glucose $<7.8 \mathrm{mmol} / \mathrm{l}(<140 \mathrm{mg} / \mathrm{dl})$

The pathogenesis of glycaemic abnormalities in $\beta$-TM is complex and multifactorial. It has been predominantly attributed to a combination of reduced insulin secretory capacity and insulin resistance. These patients are a very heterogenous group with some individuals exhibiting mainly insulin deficiency and others predominantly insulin resistance. The traditional concept has been that the initial insult is insulin resistance compensated by hyperinsulinaemia. Then, pancreatic damage and insulin deficiency subsequently develops leading to DM. However, this is not always the sequence of events leading to development of DM. It has been shown that a defect in $\beta$-cell insulin secretion can be present early before the development of glucose intolerance, resulting from toxic effects of iron deposition in the pancreas. ${ }^{20}$ Overall, the interplay between liver siderosis, causing insulin resistance and pancreatic $\beta$-cell siderosis, causing insulin deficiency, leads to the development of diabetes. Also other factors play an important role such as hepatitis $\mathrm{C}$ viral infection, autoimmunity, family history of diabetes mellitus and genetic factors. ${ }^{21}$

According to the UK standards for the clinical care of children and adults with thalassaemia published in 2008, all patients should be screened with 0GTT annually from puberty or from the age of 10 years if there is a family history of DM. Annual screening becomes even more 
important in the light of evidence showing that intensive combined chelation regimen in the early stages of glucose abnormalities can improve insulin secretion and normalise glucose metabolism. ${ }^{17}$

Diagnosis of IFG or IGT indicates a pre-diabetic state which, if not managed appropriately, will progress to diabetes. Management of these patients should include intensive chelation therapy as well as lifestyle modification. Lifestyle modification in the form of regular exercise (for example walking 150 minutes/week) and weight loss of $5-10 \%$ of body weight has been shown to reduce drastically the progression of pre-diabetes to diabetes in the general population.

Management of DM should be individualised. The $1^{\text {st }}$ line treatment in most patients should be, besides lifestyle modification, oral antidiabetic agents. There is very limited published data on the efficacy and safety of these agents in patients with TM. The only drugs used in small studies in this context with good effect are metformin, ${ }^{22}$ glibenclamide $^{23,24}$ and acarbose. ${ }^{25}$ Modern hypoglycaemic agents such as glitazones (insulin-sensitising agents), DPP-IV inhibitors (increasing intrinsic GLP-1 levels), injectable GLP-1 agonists (mediating glucosedependent insulin secretion) and SGLT-2 inhibitors (increasing renal excretion of glucose) have not been studied in patients with TM, but are increasingly becoming the mainstay of treatment of DM in the general population because of low rate of hypoglycaemias and weight loss. Some patients will need insulin therapy.

Their glycaemic control should be assessed by periodical fructosamine estimation (with fructosamine target $<322 \mathrm{umol} /$ equivalent to Hbalc $<7.0 \%$ ). They should perform home capillary blood glucose monitoring with a frequency based on their treatment.

All patients with DM should be monitored regularly for the development of complications. Their monitoring, on at least annual basis, should include:

- Measurement of urine albumin creatinine ratio

- Assessment of renal function

- Retinal screening

- Foot examination

- Measurement of blood pressure (BP target $<130 / 80 \mathrm{mmHg}$ )

- Lipid profile with targets for total cholesterol $<4.0 \mathrm{mmol} /(<155$ $\mathrm{mg} / \mathrm{dl})$ and $\mathrm{LDL}<2.0 \mathrm{mmol} / \mathrm{l}(<77 \mathrm{mg} / \mathrm{dl})$

It is worth emphasising that patients with TM and diabetes can develop diabetes-related complications. Microvascular complications include diabetic nephropathy, retinopathy and neuropathy. Limited data exist about the frequency of these complications, for example the rate of diabetic retinopathy has been reported $13.6-26.0 \%{ }^{21,26} \mathrm{~A}$ prevalence of microalbuminuria of $13.2-55.0 \%{ }^{21,27}$ has been recorded. With regards to macrovascular complications of diabetes, they include ischaemic heart disease, cerebrovascular disease and peripheral vascular disease. Recent study by Pepe et al. showed that DM in patients with TM significantly increases the risk for cardiac complications, heart failure, hyperkinetic arrhythmias and myocardial fibrosis. ${ }^{28}$

\section{Gonadal axis}

Delayed puberty and hypogonadism are the most common endocrine complications of iron overload. Hypogonadism in adult thalassaemic patients is very common with prevalence of $40-60 \%$. Hypogonadism can be either primary gonadal failure as a result of iron overload in the gonads or, more commonly, secondary (hypogonadotrophic) hypogonadism due to haemosiderosis of gonadotroph cells in the anterior pituitary gland.

In adult eugonadal thalassaemic patients, annual screening for development of hypogonadism should be performed. This should include history (libido, erectile function, frequency of spontaneous erections in males and frequency of menstrual cycles, vasomotor symptoms, libido in females), physical examination (secondary sexual characteristics including examination of genitalia in males) and biochemical assessment (testosterone, SHBG, FSH, LH in males and oestradiol, FSH, LH in females). It is worth mentioning that biochemical diagnosis of hyponadism in adult males requires measurement of serum fasting testosterone in early morning in at least 2 occasions.

Treatment of female hypogonadism aims to alleviate symptoms of oestrogen deficiency such as hot flushes, sweats, vaginal atrophy and mood changes and prevent long-term complications such as osteoporosis. Treatment is in the form of hormone replacement therapy (HRT) and should continue at least until the age of 50, the mean age of natural menopause.

Treatment of male hypogonadism aims to improve libido and erectile function, to improve mood and well-being, to improve muscle mass and strength and to improve bone mineral density by inhibiting bone resorption. Treatment is in the form of testosterone replacement therapy in a variety of preparations such as daily application of transdermal gel or IM Nebido injections every 10-14 weeks.

\section{Growth hormone axis}

Short stature and delay of growth are common features in TM with growth hormone (GH) deficiency being one of the main causes. Treatment of children with TM and reduced $\mathrm{GH}$ reserve with recombinant GH has been shown to be effective in increasing significantly linear growth velocity. However, information on treatment of growth hormone deficiency (GHD) in adult thalassaemic patients is lacking.

A study, reassessing the GH - IGF-1 axis, after attainment of final height, in 16 young adults with TM and childhood-onset GHD, found that $19 \%$ had persisting $\mathrm{GH}$ deficiency. ${ }^{29}$ For this reason, the $\mathrm{GH}$ status should be reassessed in adult TM patients with childhoodonset GHD.

Cross-sectional study on the prevalence of GHD in 94 adult thalassaemic patients with mean age 31.5 years demonstrated severe GHD in $22.3 \%$ and partial GHD in $19.1 \%$ of patients. ${ }^{30}$ All patients underwent GHRH plus arginine test; severe GHD was defined by GH peak $<9$ mcg/l, while partial GHD was defined as peak $\mathrm{GH}<9-16.5 \mathrm{mcg} / \mathrm{l}$. A similar study of 28 adult thalassaemic patients found that $32.1 \%$ had severe $\mathrm{GHD}$, defined as peak $\mathrm{GH}<11.0 \mathrm{mcg} / \mathrm{l}$ for $\mathrm{BMI}<25$ and peak $\mathrm{GH}$ $<8.0 \mathrm{mcg} /$ l for BMI>25. ${ }^{31}$ The lack of correlation between ferritin levels and $\mathrm{GH}$ peaks indicates that iron overload is probably not the only determinant in the pathogenesis of GHD in TM. In addition to iron deposition in anterior pituitary, other mechanisms such as cell damage deriving from tissue hypoxia may be relevant. Another similar study found that $25 \%$ of adult thalassaemic patients had severe GHD, while $69 \%$ of patients had serum IGF-1 values below the $95 \%$ confidence interval for age. ${ }^{32}$

All these studies showed also a very high proportion of patients with IGF-1 deficiency which could not be explained by GHD alone. Noted that GH acts either directly or indirectly through IGF-1. Plausible mechanism are $\mathrm{GH}$ resistance attributed to $\mathrm{GH}$ receptor alterations or postreceptor defects and reduced liver synthetic activity which contribute to greatly impaired IGF-1 production in patients with TM. In total, a variety of abnormalities has been described in a rage proportion of thalassaemic patients, including classic GHD with low spontaneous $\mathrm{GH}$ secretion and impaired response to $\mathrm{GH}$-releasing hormone; $\mathrm{GH}$ resistance with reduced IGF-1 response on IGF-1 generation test after $\mathrm{GH}$ administration; a combination of classic GHD and GH resistance..$^{33}$

$\mathrm{GH}$ can have an important impact on the quality of life and sense of wellbeing. GH plays an important role in lipid and glucose metabolism and also has an anabolic effect on body composition and bone mass. $\mathrm{GH}$ 
deficiency in adults may lead to numerous sequalae, such as reduced quality of life and especially reduced energy levels; reduced bone mineral density (osteopenia / osteoporosis); altered body composition (reduced lean mass, increased fat mass, truncal obesity); reduced muscle strength and exercise capacity; hyperlipidaemia with high levels of LDL; increased thickness of intima media of blood vessels, impaired ventricular contractility and cardiovascular morbidity. On the one hand, treatment with GH may improve quality of life and psychological wellbeing, increase lean body mass and reduce fat mass, increase bone mineral density, reduce total cholesterol by around $15 \%$ and improve ventricular function. On the other hand, treatment with recombinant GH can have side effects, such as deterioration of glycaemic control and development of diabetes due to insulin resistance, fluid retention with peripheral oedema, headaches, arthralgias and myalgias.

The studies above confirm the necessity for assessment of $\mathrm{GH}$ reserve in adult thalassaemic patients in the form of serum IGF-1 levels in combination with GHRH - arginine test. Future studies are warranted to establish the effectiveness of recombinant $\mathrm{GH}$ replacement in adult thalassaemic patients with GHD. The potential clinical benefits of $\mathrm{GH}$ replacement in these patients remains to be determined.

\section{Adrenals}

Adrenal insufficiency, manifested as adrenal crisis, appears to be extremely rare in thalassaemic patients. However, several recent studies have reported significant prevalence of "biochemical" adrenal insufficiency, ranging from $18-45 \%$. A recent study, including 56 thalassaemic children and adolescents, showed that $37.5 \%$ of participants had adrenal insufficiency confirmed in ITT, the gold standard test. ${ }^{34}$ This study was unique for two important reasons; first, this was the only study using ITT, considered as the gold standard for the diagnosis of adrenal insufficiency, and second, demonstrated that cortisol binding globulin (CBG) levels were normal, indicating serum total cortisol levels were a good surrogate marker of serum free "active" cortisol. In total, $19.7 \%$ of patients had after ITT peak serum cortisol $<14 \mathrm{mcg} / \mathrm{dl}$ and they should be strongly considered for regular hydrocortisone replacement, while $17.8 \%$ had peak serum cortisol $14-20 \mathrm{mcg} / \mathrm{dl}$ and would need steroid cover for major illnesses or operations. ${ }^{34}$ This study concluded that all thalassaemic patients should have $1 \mathrm{mcg}$ cosyntropin (synthetic ACTH) test as an adrenal function screening test. In the light of $1 \mathrm{mcg}$ cosyntropin test being false positive in $18 \%$ of cases, for definite diagnosis, ITT should be performed in those having serum peak cortisol value $<16 \mathrm{mcg} / \mathrm{dl}$ after $1 \mathrm{mcg}$ cosyntropin test. ${ }^{34}$ Another study of 124 adult patients with $\beta$-TM major demonstrated a subnormal cortisol response after $1 \mathrm{mcg}$ cosyntropin test in $32.1 \%$ of patients. It is worth mentioning that only $1.7 \%$ of patients would qualify for regular hydrocortisone replacement, while the remaining $30.4 \%$ of patients would potentially benefit from steroid cover during major illnesses and preoperatively. Most patients in this cohort exhibited a subtle impairment of adrenocortical function which may become clinically relevant in case of major stressful events. ${ }^{35}$ Another recent study of 23 thalassaemic patients ( 10 children and 13 young adults) showed a prevalence of $34.7 \%$ for adrenal insufficiency, based on peak cortisol value $<20$ $\mathrm{mcg} / \mathrm{dl}$ and increment $<7 \mathrm{mcg} / \mathrm{dl}$ after $1 \mathrm{mcg}$ cosyntropin. The same study showed a much lower rate of adrenal insufficiency of $8.7 \%$, using the same cut-off values after $250 \mathrm{mcg}$ cosyntropin. ${ }^{36}$

In conclusion, several studies have suggested high prevalence of partial adrenal insufficiency. Therefore, patients with $\beta$-TM major need to have regular evaluation of hypothalamus - pituitary - adrenal axis. Steroid cover should be strongly considered in these patients during major physical or surgical stress. Interestingly, all these patients are considered 'asymptomatic', but manifestations of mild glucocorticoid defi- ciency may be masked by symptoms commonly reported such as fatigue, lack of energy, arthralgias, lack of appetite and muscle weakness.

\section{Thyroid}

Frequency of hypothyroidism in patients with TM major has been reported between $2 \%$ and $30 \%$. Hypothyroidism is mainly attributed to iron overload, while thyroid autoimmunity has no role in the pathogenesis of hypothyroidism in b-TM. The much lower prevalence of antithyroid antibodies in patients with TM than in age-matched euthyroid women suggests that iron overload may inhibit thyroid autoimmunity. ${ }^{37}$

- Hypothyroidism can be classified into:

Subclinical (normal fT4 and elevated TSH 5-10 mU/ml) which usually does not require thyroxine replacement.

Subclinical hypothyroidism (normal fT4 and elevated TSH > 10 $\mathrm{mU} / \mathrm{ml}$ ) when thyroxine replacement should be considered.

- Overt hypothyroidism (low fT4 and elevated TSH) when thyroxine replacement should be initiated.

Central hypothyroidism has been traditionally regarded uncommon. However, a recent longitudinal study of 48 TM major patients over a 12year period reported that $13 / 48$ patients developed hypothyroidism without appropriate increase in TSH levels; this suggested a progressive slow dysfunction of thyroid gland with a degree of pituitary insensitivity to low fT4 levels (central component of hypothyroidism). ${ }^{38}$

Progression is variable and slow; it may take years to decades to progress from normal thyroid status to overt hypothyroidism. ${ }^{39}$ Recent data has suggested that intensive iron chelation regimens can reverse thyroid dysfunction, especially I the early subclinical stages. In cases of overt hypothyroidism, the impairment of thyroid dysfunction is usually permanent and irreversible. ${ }^{40}$

Thyroid function should be assessed annually with measurement of serum free T4 and TSH from the age of 9 or earlier if there is a clinical suspicion on hypothyroidism.

\section{Parathyroids}

The prevalence of over hypoparathyroidism varies between 1.2 and $19.0 \%$, while subtle subclinical abnormalities in parathyroid hormone (PTH) secretion are much more common with limited clinical significance. The majority of patients with clinical hypoparathyroidism have a mild form of the disease with paraesthesia being the only clinical manifestation. In rare occasions, patients can develop more severe form of hypoparathyroidism and present with tetany and seizures. The variety of clinical manifestations of hypocalcaemia are mainly due to increase of neuromuscular excitability. These include tingling of fingers, toes or lips; numbness of fingers, toes or lips; cramps; carpopedal spasm; stridor due to laryngospasm and seizures.

All patients should have annual screening for hypoparathyroidism from the age of 16, including plasma calcium, phosphate and PTH levels. Low plasma calcium levels in combination with high phosphate and low or even inappropriate normal PTH levels indicates hypoparathyroidism.

Treatment of hypoparathyroidism aims not to achieve normalisation of plasma calcium levels, but to render the patient asymptomatic with a plasma calcium at the lower end of normal range or even just below the normal lower cut-off. The reason for this is that the renal retention of calcium mediated by PTH has been lost. Therefore, any attempt to raise plasma calcium well into the normal range is likely to result in hypercalciuria with the associated risks of renal stones and nephrocalcinosis.

Treatment of hypoparathyroidism contains:

- Hydroxylated vitamin D derivatives. Vitamin D requires 1a-hydroxyla- 
tion by the kidney to become active. In hypoparathyroidism, there is deficiency of PTH which is essential for la-hydroxylation of vitamin D. The hydroxylated vitamin D analogues are either la-hydroxycholecalciferol ( $\alpha$ calcidol) at an initial dose of $0.25-0.5 \mathrm{mcg}$ orally once per day or 1,25-dihydroxycholecalciferol (calcitriol) at an initial dose of $0.25 \mathrm{mcg}$ orally twice per day. In the initial stages after initiation of treatment, plasma calcium and phosphate levels should be monitored closely (at least weekly) in order to avoid hypercalcaemia. The same applies to every change in the dose of vitamin D analogues with measurement of plasma calcium at least 1 week after. After establishing the appropriate maintenance treatment dose which achieves target calcium at the lower end of normal range, plasma calcium levels should be measured every 3 months. The usual maintenance dose for both vitamin D analogues are $0.25-1 \mathrm{mcg} /$ day.

Oral calcium supplements may be needed in some patients, especially if dietary calcium intake is inadequate. Usual does are 1-3 gr of elemental calcium per day by mouth.

\section{References}

1. Ang AL, Tzoulis P, Prescott E, et al. History of myocardial iron loading is a strong risk factor for diabetes mellitus and hypogonadism in adults with $\beta$ thalassemia major. European journal of haematology 2014;92:229-36.

2. Thuret I, Pondarre C, Loundou A, et al. Complications and treatment of patients with $\beta$-thalassemia in France: results of the National Registry. Haematologica 2010;95:724-9.

3. Jensen CE, Tuck SM, Old J, et al. Incidence of endocrine complications and clinical disease severity related to genotype analysis and iron overload in patients with $\beta$-thalassaemia. European journal of haematology 1997;59:76-81.

4. Sharma R, Seth A, Chandra J, et al. Endocrinopathies in adolescents with thalassaemia major receiving oral iron chelation therapy. Paediatrics and international child health 2014: 2046905514 Y0000000160.

5. Isik P, Yarali N, Tavil B, et al. Endocrinopathies in Turkish children with Beta thalassemia major: results from a single center study. Pediatric hematology and oncology 2014;31:607-15.

6. Vogiatzi MG, Macklin EA, Trachtenberg FL, et al. Differences in the prevalence of growth, endocrine and vitamin D abnormalities among the various thalassaemia syndromes in North America. British journal of haematology 2009;146:546-56.

7. De Sanctis V, Eleftheriou A, Malaventura C, Thalassaemia International Federation Study Group on G, Endocrine Complications in T. Prevalence of endocrine complications and short stature in patients with thalassaemia major: a multicenter study by the Thalassaemia International Federation (TIF). Pediatric endocrinology reviews: PER 2004;2 Suppl 2:249-55.

8. Gamberini MR, De Sanctis V, Gilli G. Hypogonadism, diabetes mellitus, hypothyroidism, hypoparathyroidism: incidence and prevalence related to iron overload and chelation therapy in patients with thalassaemia major followed from 1980 to 2007 in the Ferrara Centre. Pediatric endocrinology reviews: PER 2008;6 Suppl 1:158-69.

9 . Au WY, Lam WW, Chu WW, et al. A cross-sectional magnetic resonance imaging assessment of organ specific hemosiderosis in 180 thalassemia major patients in Hong Kong. Haematologica 2008;93:784-6.

10. Belhoul KM, Bakir ML, Saned MS, et al. Serum ferritin levels and endocrinopathy in medically treated patients with $\beta$ thalassemia major. Annals of hematology 2012;91:1107-14.

11. Chirico V, Rigoli L, Lacquaniti A, et al. Endocrinopathies, metabolic disorders, and iron overload in major and intermedia tha- lassemia: serum ferritin as diagnostic and predictive marker associated with liver and cardiac T2* MRI assessment. European journal of haematology 2014.

12. Noetzli LJ, Papudesi J, Coates TD, Wood JC. Pancreatic iron loading predicts cardiac iron loading in thalassemia major. Blood 2009;114:4021-6.

13. Noetzli LJ, Mittelman SD, Watanabe RM, et al. Pancreatic iron and glucose dysregulation in thalassemia major. American journal of hematology 2012;87:155-60.

14. Noetzli LJ, Panigrahy A, Mittelman SD, et al. Pituitary iron and volume predict hypogonadism in transfusional iron overload. American journal of hematology 2012;87:167-71.

15. Tanner MA, Galanello R, Dessi C, et al. A randomized, placebo-controlled, double-blind trial of the effect of combined therapy with deferoxamine and deferiprone on myocardial iron in thalassemia major using cardiovascular magnetic resonance. Circulation 2007;115:1876-84.

16. Farmaki K, Tzoumari I, Pappa C, et al. Normalisation of total body iron load with very intensive combined chelation reverses cardiac and endocrine complications of thalassaemia major. British journal of haematology 2010;148:466-75.

17. Farmaki K, Angelopoulos N, Anagnostopoulos G, et al. Effect of enhanced iron chelation therapy on glucose metabolism in patients with $\beta$-thalassaemia major. British journal of haematology 2006;134:438-44.

18. Casale M, Citarella S, Filosa A, et al. Endocrine function and bone disease during long-term chelation therapy with deferasirox in patients with $\beta$-thalassemia major. American journal of hematology 2014;89:1102-6.

19. Cunningham MJ, Macklin EA, Neufeld EJ, et al. Complications of $\beta$ thalassemia major in North America. Blood 2004;104:34-9.

20. Angelopoulos NG, Zervas A, Livadas S, et al. Reduced insulin secretion in normoglycaemic patients with $\beta$-thalassaemia major. Diabetic medicine : a journal of the British Diabetic Association 2006;23:1327-31.

21. Tzoulis P, Shah F, Jones R, e al. Joint diabetes thalassaemia clinic: an effective new model of care. Hemoglobin 2014;38:104-10.

22. Dhouib N, Turki Z, Mellouli F, et al. Efficacy of metformin in the treatment of diabetes mellitus complicating thalassemia major. La Tunisie medicale 2010;88:136.

23. Ladis V, Theodorides C, Palamidou F, et al. Regulation of glucose disturbances with glibenclamide in patients with thalassemia. Annals of the New York Academy of Sciences 1998;850:471-4.

24. Ladis V, Theodorides C, Palamidou F, et al. Glucose disturbances and regulation with glibenclamide in thalassemia. Journal of pediatric endocrinology \& metabolism : JPEM 1998;11 Suppl 3:871-8.

25. Mangiagli A, Campisi S, De Sanctis V, et al. Effects of acarbose in patients with $\beta$-thalassaemia major and abnormal glucose homeostasis. Pediatric endocrinology reviews: PER 2004;2 Suppl 2:276-8.

26. Incorvaia C, Parmeggiani F, Mingrone G, et al. Prevalence of retinopathy in diabetic thalassaemic patients. Journal of pediatric endocrinology \& metabolism : JPEM 1998; 11 Suppl 3:879-83.

27. Loebstein R, Lehotay DC, Luo X, et al. Diabetic nephropathy in hypertransfused patients with $\beta$-thalassemia. The role of oxidative stress. Diabetes care 1998;21:1306-9.

28. Pepe A, Meloni A, Rossi G, et al. Cardiac complications and diabetes in thalassaemia major: a large historical multicentre study. British journal of haematology 2013;163:520-7.

29. La Rosa C, De Sanctis V, Mangiagli A, et al. Growth hormone secretion in adult patients with thalassaemia. Clinical endocrinology 2005;62:667-71.

30. Scacchi M, Danesi L, Cattaneo A, et al. Growth hormone deficiency (GHD) in adult thalassaemic patients. Clinical endocrinology 2007;67:790-5. 
31. Poggi M, Pascucci C, Monti S, et al. Prevalence of growth hormone deficiency in adult polytransfused $\beta$-thalassemia patients and correlation with transfusional and chelation parameters. Journal of endocrinological investigation 2010;33:534-8.

32. Vidergor G, Goldfarb AW, Glaser B, Dresner-Pollak R. Growth hormone reserve in adult $\beta$ thalassemia patients. Endocrine 2007;31:33-7.

33. Spiliotis BE. Beta-thalassemia and normal growth: are they compatible? European journal of endocrinology / European Federation of Endocrine Societies 1998;139:143-4.

34. Poomthavorn P, Isaradisaikul B, Chuansumrit A, et al. High prevalence of "biochemical" adrenal insufficiency in thalassemics: is it a matter of different testings or decreased cortisol binding globulin? The Journal of clinical endocrinology and metabolism 2010;95:4609-15.

35. Scacchi M, Danesi L, Cattaneo A, et al. The pituitary-adrenal axis in adult thalassaemic patients. European journal of endocrinology / European Federation of Endocrine Societies 2010;162:43-8.

36. Soliman AT, Yassin M, Majuid NM, et al. Cortisol response to low dose versus standard dose (back-to-back) adrenocorticotrophic stimulation tests in children and young adults with thalassemia major. Indian journal of endocrinology and metabolism 2013;17:1046-52.

37. Mariotti S, Pigliaru F, Cocco MC, et al. $\beta$-thalassemia and thyroid failure: is there a role for thyroid autoimmunity? Pediatric endocrinology reviews: PER 2011;8 Suppl 2:307-9.

38. Soliman AT, Al Yafei F, Al-Naimi L, et al. Longitudinal study on thyroid function in patients with thalassemia major: high incidence of central hypothyroidism by 18 years. Indian journal of endocrinology and metabolism 2013;17:1090-5.

39. Landau H, Matoth I, Landau-Cordova Z, et al. Cross-sectional and longitudinal study of the pituitary-thyroid axis in patients with thalassaemia major. Clinical endocrinology 1993;38:55-61.

40. Chirico V, Lacquaniti A, Salpietro V, et al. Thyroid dysfunction in thalassaemic patients: ferritin as a prognostic marker and combined iron chelators as an ideal therapy. European journal of endocrinology / European Federation of Endocrine Societies 2013;169:785-93. 